\title{
An Estimate of the Incidence of Depression in Idiopathic Parkinson's Disease
}

\author{
George Dooneief, MD; Elizabeth Mirabello, MD; Karen Bell, MD; Karen Marder, MD; \\ Yaakov Stern, PhD; Richard Mayeux, MD
}

\begin{abstract}
- Estimates of the prevalence of depression in idiopathic Parkinson's disease vary, but have been greater than in most comparison groups. In a survey of patients with Parkinson's disease $(N=339)$, the prevalence of depression was $47 \%$. A total of 326 cases were reviewed to estimate the incidence of depression from September 30, 1984, to July 31, 1989. Assessments of depression during both the prevalent and the incident periods were noted in $\mathbf{2 5 8}$ cases. There was no history of depression in 129 cases, and nine new cases occurred. The incidence rate was $1.86 \%$ per year and the cumulative risk was 8.6\%. Published estimates of the incidence of depression in the general population are few. In one study, the annual incidence of depression in individuals older than 40 years was $0.17 \%$. In another, the incidence of depression in individuals older than 50 years was $0.14 \%$ for men and $0.29 \%$ for women. Although our retrospective study probably underdiagnoses depression, the incidence of depression is increased in Parkinson's disease.
\end{abstract}

(Arch Neurol. 1992;49:305-307)

$\mathbf{E}$ stimates of the prevalence of depression in idiopathic Parkinson's disease (PD) vary, but have been greater than in most comparison groups. ${ }^{1}$ The prevalence of depression is a function of the incidence of the new onset, duration, and recurrence rate of depressive episodes. We theorized that an estimate of the incidence, rather than the prevalence, would provide a better measure of the risk of depression in PD. In a survey of patients with PD that was completed on September 30,1984 , we noted the prevalence of depression to be $47 \% .^{2}$ The current study estimates the incidence and the cumulative risk of depression in the same cohort of patients with PD based on a second review of the medical records 4 years and 10 months later.

\section{SUBJECTS AND METHODS Subjects}

All patient-records were obtained from the ColumbiaPresbyterian Medical Center (CPMC), New York, NY. The medical center functions as both a tertiary referral center and a community hospital for northern Manhattan. The records of all patients with parkinsonism seen at CPMC between March 1, 1983, and September $30,1984(\mathrm{~N}=442)$, were reviewed. Three hundred thirty-nine of the patients were judged to have idiopathic $\mathrm{PD}$ with onset at the age of 40 years or older and were included in a prior study. ${ }^{2}$ No new patients were entered into the cohort.

\section{Data Extraction}

All records were reviewed to determine the presence or absence of depression from September 30, 1984, to July 31, 1989,

\footnotetext{
Accepted for publication September 23, 1991.

From the Departments of Neurology (Drs Dooneief, Mirabello, Bell, Marder, Stern, and Mayeux) and Psychiatry (Drs Stern and Mayeux), College of Physicians and Surgeons, Columbia University, New York, NY.

Reprint requests to Columbia University, Sergievsky Center, 630 W 168th St, New York, NY 10032 (Dr Mayeux).
}

a period of 4 years and 10 months. A data extraction form was developed for the process of record review. The date when depression was first diagnosed and the date of the last chart entry were recorded. When the date of the onset of depression was unclear, we used the midpoint between September 30, 1984, and the date of the last chart entry as the estimated date of onset.

Subjects not examined at the medical center after September 30 , 1984, were tracked and telephoned. These subjects, or their responsible family member, were questioned using a structured interview to obtain information identical to that on the data extraction form. For these cases, the date of last entry was July 31, 1989.

\section{Diagnostic Criteria}

We used criteria for depression modified from the Diagnostic and Statistical Manual of Mental Disorders, Revised Third Edition. ${ }^{3} \mathrm{~A}$ unified Parkinson's disease rating scale ${ }^{4}$ score of 3 or more on the depression item was accepted as diagnostic. According to this scale, 0 indicates no depression; 1 , periods of sadness or guilt, never sustained for days or weeks; 2 , sustained depression (1 week or longer); 3 , sustained depression with vegetative symptoms (insomnia, anorexia, weight loss, loss of interest); and 4, sustained depression with vegetative symptoms and suicidal thoughts or intent. Otherwise, depression was defined as a persistently depressed mood (not a grief reaction, eg, following the death of a spouse) or a feeling of sadness or being "down-inthe-dumps" associated with a loss of interest in usual activities and with vegetative signs and symptoms.

For the diagnosis of depression, the following guide was used to assess the quality of diagnostic assurance:

1. Definite. A neurologist or psychiatrist made the diagnosis and recorded the information in the chart.

2. Probable. All of the signs and symptoms were present, but a "qualified" examiner had not written the diagnosis in the record.

3. Possible. Symptoms and signs were mentioned by nurses, social workers, or other health professionals, but no diagnosis had been made. For telephone interviews, the diagnosis of depression was always considered "possible" unless we spoke to the patient's physician.

Only first episodes of depression were considered.

\section{Data Analysis}

Incidence rates, cumulative risk, and standardized morbidity ratios (SMRs) were calculated as described by Rothman. ${ }^{5}$ The SMRs were computed in comparison with age- and sex-specific rates from the studies by Essen-Moller and Hagnell, ${ }^{6}$ Hagnell et al, ${ }^{7}$ and Murphy et al. ${ }^{8}$

\section{RESULTS}

The Figure outlines the flow of the original cohort; $339 \mathrm{pa}$ tients with PD were considered eligible for this review. No records were available for 13 . Two had not been rated on the depression item, and 14 were rated "don't know" during the initial survey. Of the remaining 310,41 had no rating of depression during the incident period, as there was no new information in the medical records and we were unable to locate the patient or family. An additional 11 were rated "don't know" during the incident period. This left 258 records for 


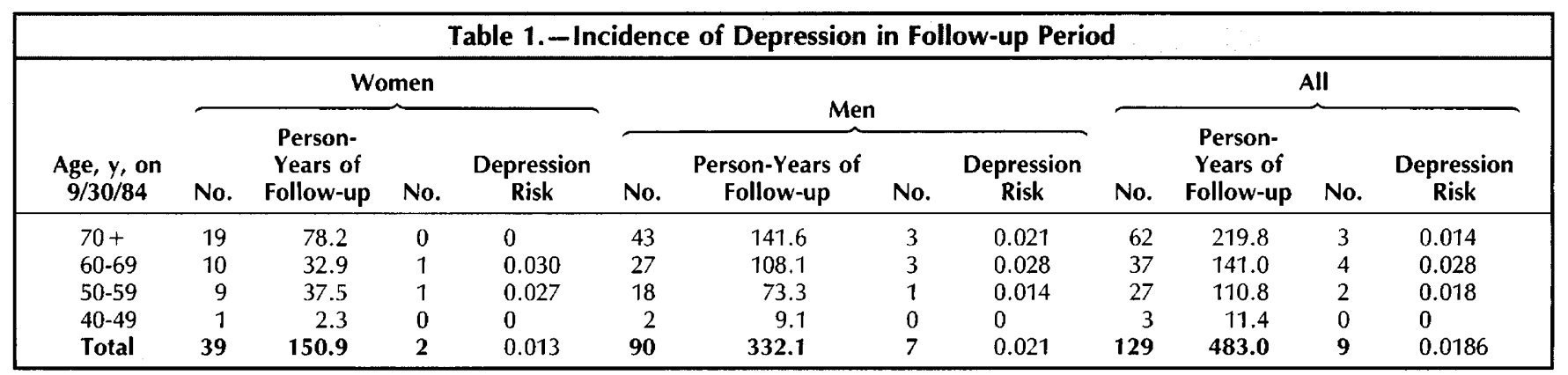

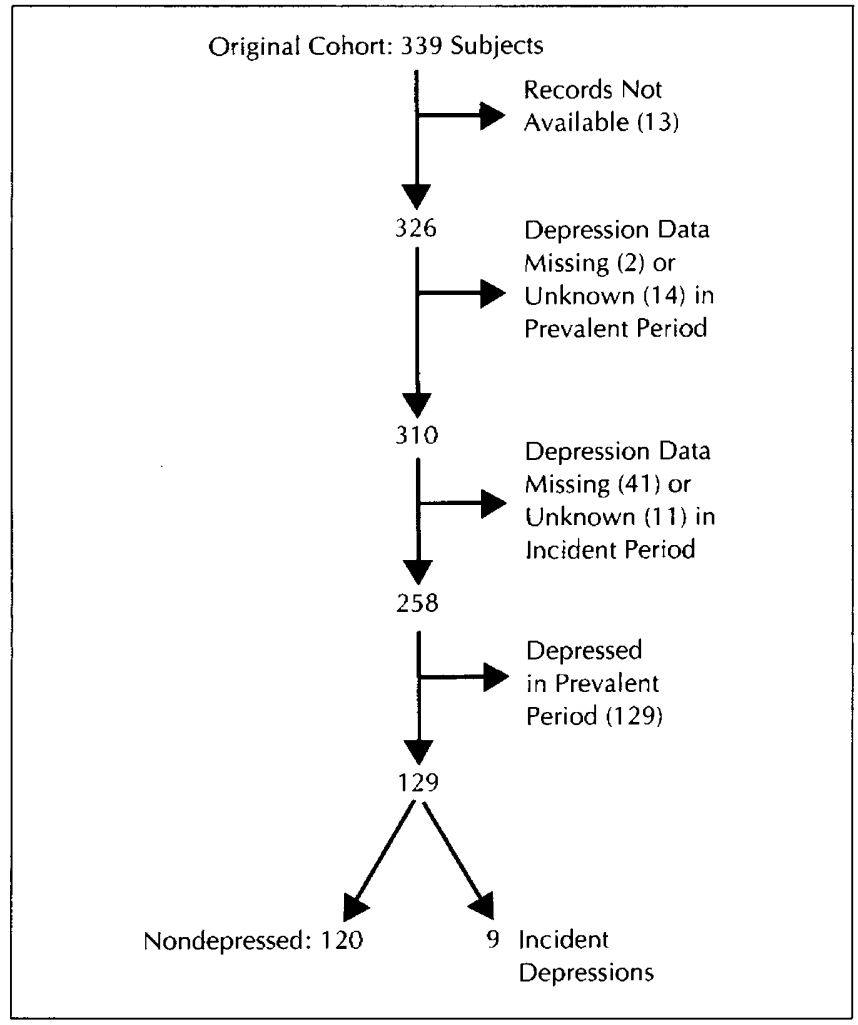

Flow chart for incidence study.

review; 129 of these patients had been considered depressed in the previous study. Therefore, we began our review with the records of 129 patients who had not previously been depressed. There were $39(30 \%)$ women and $90(70 \%)$ men (proportions similar to those in the original cohort [127 women and $199 \mathrm{men}])$. The mean age of this group at last chart entry was $71.5 \pm 9.3$ years.

After review of the 129 patient-records, 120 of the patients still did not demonstrate evidence of depression. First onset of depression was noted in nine patients. In seven cases, depression was clearly defined and the approximate date of onset was noted in the record. In two patients, the onset of depression clearly occurred after September 30, 1984, but t'ie date of onset could not be established. The diagnostic certainty was definite in seven cases, probable in one case, and possible in one. The aggregate follow-up for the 129 patients from the end of the prevalent period until either the last chart entry or the date of onset of depression was 483 personyears. The incidence rate of depression in this cohort was therefore $1.86 \%$ per year, with $95 \%$ confidence interval from $1.16 \%$ to $2.56 \%$ (Table 1 ). The cumulative risk for depression was $8.6 \%$ during this period.

\begin{tabular}{|lcc|}
\hline \multicolumn{3}{|c|}{ Table 2. $\begin{array}{c}\text { Comparison of Nondepressed and } \\
\text { Depressed Patients* }\end{array}$} \\
\hline & Nondepressed & Depressed \\
\hline No. & 120 & 9 \\
Male:female (ratio) & $83: 37(2.2)$ & $7: 2(3.5)$ \\
In-patient & $12(10 \%)$ & $1(11 \%)$ \\
Age, $y$ & $71.6 \pm 9.4$ & $70.0 \pm 6.1$ \\
Age at onset of PD, y & $58.8 \pm 11.0$ & $58.7 \pm 9.7$ \\
Duration of disease, y & $8.1 \pm 6.6$ & $6.8 \pm 4.2$ \\
Duration of levodopa & $5.8 \pm 4.6$ & $5.4 \pm 4.7$ \\
therapy, y & $20(16 \%)$ & $1(11 \%)$ \\
Family history of PD & $90(87 \%)$ & $6(86 \%) \dagger$ \\
Responders to levodopa & $21(18 \%)$ & $2(22 \%)$ \\
Used dopa agonists & $79(66 \%)$ & $6(67 \%)$ \\
Tremulous & $67.6 \pm 33.9$ & $71.1 \pm 27.5$ \\
ADL (on), \% & $38.8 \pm 40.1$ & $33.3 \pm 35.1$ \\
ADL (off), $\%$ & $12(11 \%)$ & $2(22 \%)$ \\
Benign course & $101(89 \%)$ & $6(67 \%)$ \\
Average course & 0 & $1(11 \%)$ \\
Rapid course & & \\
\hline
\end{tabular}

PD indicates Parkinson's disease; $A D L$, activities of daily living rating.

tData missing for two patients.

As shown in Table 2, the group that became depressed and the group that remained nondepressed did not differ significantly with respect to age, sex, age at onset of PD, duration of levodopa therapy, response to levodopa therapy, use of dopa agonists, family history of PD, presence of tremor, or impairment of activities of daily living rating (on the Schwab and England ${ }^{9}$ activities of daily living scale). The course of illness (rapid, average, or benign) did not predict depression. The average duration of PD was shorter in the depressed group, but this difference did not reach statistical significance.

Age- and sex-specific SMRs for depression incidence rates revealed an overall SMR of 41.5; ie, depression was found 41.5 times more frequently in the patients with PD than would be expected in a general population, using the population study of Essen-Moller and Hagnell ${ }^{6}$ to generate expected rates of depression. When data from the extension of this study were used, ${ }^{7}$ the overall SMR fell to 10.3. Finally, the use of the sex-specific rates from the study by Murphy et $\mathrm{al}^{8}$ revealed an SMR for depression incidence of 3.8 in women older than 50 years and an SMR of 148.9 for men older than 50 years. 


\section{COMMENT}

Depression is probably the most common mental disturbance in PD. ${ }^{1}$ Forty-seven percent of the patients with PD who we reviewed were depressed during the initial survey. ${ }^{2}$ In a review of 14 studies that included more than 1500 patients, Gotham et a ${ }^{10}$ estimated the mean prevalence of depression in PD to be $46 \%$, although prevalence ranged from $20 \%$ to as high as $90 \%$ in these studies. Different sampling and assessment techniques may have accounted for much of the variation. In the healthy elderly population, Boyd and Weissman, "in their review, noted that the point prevalence of depression was $3 \%$ for men and $4 \%$ for women. This certainly suggests that depression is more prevalent in individuals with PD than in the healthy elderly.

While the prevalence of depression has been described, and is higher in patients with PD than in age-matched controls, the incidence of depression in PD is unknown. In fact, the incidence of nonbipolar depression has rarely been reported. Boyd and Weissman ${ }^{\mathrm{I1}}$ concluded that the lifetime risk for depression was $8 \%$ to $12 \%$ for men and $20 \%$ to $26 \%$ for women, but they felt that they could not confidently estimate the incidence of depression from 12 studies reporting this statistic. Nine were case-registry studies, and the three longitudinal studies used varying diagnostic methods. The incidence rates ranged from $0.13 \%$ per year to $7.8 \%$ per year. Our figure of $1.86 \%$ per year fell within this broad range.

In a study that employed an analysis of data from interviews with probands and their relatives and hospital records in a small rural district of Scania (the Lundby study), ${ }^{6}$ the prevalence of depression in the adult population was $3.7 \%$ for women and $2.1 \%$ for men in 1947 . A rereview of nearly $99 \%$ of the original cohort in 1957 yielded 15 new cases of depression among those who were older than 40 years in 1947 during an aggregate follow-up of 8931 person-years-equivalent to an incidence rate of $0.17 \%$ per year. An additional review of this cohort, conducted in a similar fashion, revealed 104 new cases in the period from 1957 to 1972 (with an aggregate follow-up of 15404.4 person-years) among those older than 40 years in 1957, yielding an incidence rate of $0.68 \%$ per year. ${ }^{7}$ Murphy et $\mathrm{al}^{8}$ reported an incidence of depression of $0.14 \%$ per year for men older than 50 and an incidence of $0.29 \%$ per year for women older than 50 in a follow-up of a cohort of previously nondepressed subjects first interviewed in 1952 in the Stirling county study. ${ }^{8}$

Both the Lundby and the Stirling county studies were based primarily on interviews and were performed prospectively. Our study was a retrospective chart review. Cases might have been missed in our study, since the appropriate information might not have been sought or systematically recorded at the time of the examinations. As a result, our figures probably underestimate the incidence of depression in this cohort of patients with PD. Even so, the SMRs calculated with respect to the Lundby and Stirling county studies suggest that the incidence of depression is considerably higher in individuals with PD than in general populations of similar ages.

While there are no other studies reporting the incidence of depression in PD, Brown et $\mathrm{al}^{12}$ in their follow-up of 132 patients with PD noted that 15 of 96 previously nondepressed patients became depressed (on the basis of self-rating on the Beck Depression Inventory ${ }^{13}$ ) during a follow-up period with a median of 14 months. While they did not report an incidence rate, assuming a mean (rather than median) follow-up of 14 months among the 81 nondepressed patients, and that the 15 newly depressed patients had an onset of depression at an average of half the median follow-up period ( 7 months), then the aggregate follow-up for this cohort would be 103.5 person-years and an estimated incidence of depression would be $14.5 \%$ per year. This figure, however, must be regarded as a very rough approximation, given the nature of the method used to calculate it. Comparability with our data suffers from the differing criteria used (the Beck Depression Inventory vs the Diagnostic and Statistical Manual of Mental Disorders-Revised Third Edition), as well as from differences in the method of data collection (self-report vs chart review). However, Brown and colleagues' data also suggest a high incidence of depression in PD.

Our study is limited in many respects. Review of records may be insufficient to reliably assess the presence of depression, since the data are not always specifically asked for in routine visits. Psychiatric interviews were not performed nor were any depression measures administered systematically to this cohort. There was no "internal" comparison group reviewed in a similar fashion that could be used as controls. However, we believe that these factors would tend to produce an underestimate of the incidence of depression in $\mathrm{PD}$ and that our figures are therefore probably conservative estimates. The utility of these data is that they allow the clinician to predict about an $8 \%$ to $9 \%$ chance of patients with PD developing depression over a 5-year period. A better estimate of the morbidity due to depression in PD awaits the completion of a prospective longitudinal follow-up (including psychiatric assessment) of a large cohort of patients with PD.

This study was supported by federal grants AG-07232 and AG02802, the Alzheimer's Disease Research Center, New York, NY (AG 08702), and the Parkinson's Disease Foundation of New York (NY).

\section{References}

1. Mayeux R. A current analysis of behavioral problems in patients with idiopathic Parkinson's disease. Mov Disord. 1989;4(suppl 1): $\$ 48-\$ 56$.

2. Mayeux R, Stern Y, Rosenstein R, et al. An estimate of the prevalence of dementia in idiopathic Parkinson's disease. Arch Neurol. $1988 ; 45: 260-262$

3. American Psychiatric Association. Committee on Nomenclature and Statistics. Diagnostic and Statistical Manual of Mental Disorders-Revised Third Edition. Washington, DC: American Psychiatric Association; 1980.

4. Lesser RP, Fahn S, Snider SR, et al. Analysis of the clinical problems in parkinsonism and the complications of long-term levodopa therapy. Neurology. 1979;29:1253-1260.

5. Rothman KJ. Modern Epidemiology. Boston, Mass: Little Brown \& Co Inc; 1986:29-34, 227-233.

6. Essen-Molier E, Hagnell $O$. The frequency and risk of depression within a rural population group in Scania. Acta Psychiatr Scand Suppl. $1961 ; 162: 28-32$.

7. Hagnell O, Lanke J, Rorsman B, Ojesjo L. Are we entering an age of melancholy? depressive illnesses in a prospective epidemiological study over 25 years: the Lundby study, Sweden. Psychol Med. 1982;12:279-289.

8. Murphy I, Olivier D, Monson R, Sobol A, Leighton A. Incidence of depression and anxiety: the Stirling county study. Am J Public Health. $1988 ; 78: 534-540$.

9. Schwab JF, England AC. Projection technique for evaluating surgery in Parkinson's disease. In: Gilliam FJ, Donaldson MC, eds. Third Symposium on Parkinson's Disease. Edinburgh, Scotland: E \& S Livingstone; 1969:152-157.

10. Gotham AM, Brown RG, Marsden CD. Depression in Parkinson's disease: a quantitative and qualitative analysis. INeurol Neurosurg Psychiatry. 1986;49:381-389.

11. Boyd $\mathrm{JH}$, Weissman MM. Epidemiology of affective disorders. Arch Gen Psychiatry. 1981;38:1039-1046.

12. Brown RG, MacCarthy B, Gotham AM, Der GJ, Marsden CD. Depression and disability in Parkinson's disease: a follow-up of 132 cases. Psychol Med. 1988;18:49-55.

13. Beck AT, Ward Ch, Mendelson M, Mock J, Erbaugh J. An inventory for measuring depression. Arch Gen Psychiatry. 1961; 4:561-567. 